\title{
Fabrication of aligned carbon nanotube-filled rubber composite
}

Yoong Ahm Kim, Takuaya Hayashi, and Morinobu Endo

Faculty of Engineering, Shinshu University, 4-17-1 Wakasato, Nagano-shi 380-8553, Japan

Yasuo Gotoh

Faculty of Textile Engineering, Shinshu University, 3-15-1 Tokida, Ueda, Nagano 386-8567, Japan

Noriaki Wada, Junji Seiyama

Bando Chemical Industries, LTD. 3-1-6 Ashihara-Dori, Hyog-ku, Kobe 652-0882, Japan

\begin{abstract}
Here we fabricated rubber composite sheets filled with $5 \mathrm{wt} \%$ and $30 \mathrm{wt} \%$ of highly aligned carbon nanotubes (CNTs) through conventional rubber technology. The alignment of CNTs was possibly derived from dragged shear force during the optimized milling process. The selective alignment of CNTs leaded to enhancement of elastic modulus, thermal conductivity, electrical conductivity, and electromagnetic shielding property compared to neat rubber sheet.
\end{abstract}

Keywords: Blending; Carbon Nanotubes; Rubber; Alignment; Nanocomposite,

\section{Introduction}

Great deal of attention has been paid on tiny but fascinating carbon nanotubes consisting of enrolled graphene sheet built from $s p^{2}$ carbon units [1-3] because they have been considered as an ideal reinforcing filler in a wide range of composite systems [4], due to their long macro-morphology (high aspect ratio, length/diameter) and their exceptional mechanical property (Young's modulus $=1-1.8 \mathrm{TPa}$ ) [5], transport conductivity (resistivities at 300K of $\sim 1.2 \times 10-4-5.1 \times 10-6 \Omega \cdot \mathrm{cm}$; activation energies $<300 \mathrm{meV}$ for semiconducting tubes) $[6,7]$, thermal conductivity $(3000 \mathrm{~W} / \mathrm{m}-\mathrm{K})$.

At present, large quantity of carbon nanotubes is available caused by the recent progress of synthetic method $[8,9]$. When considering practical application of carbon nanotubes, the largest anticipated large-consumption of carbon nanotubes is as a filler for various polymers, when exploiting the excellent mechanical and electrical properties of carbon nanotubes [1]. In this sense, carbon nanotubes have been investigated as functional filler in polymer [10]. In order to 
exploit intrinsic properties of carbon nanotubes in composite, two basic problems have to be solved: dispersion and alignment. In this study, we judiciously selected commercial ethylene-prolyene-diene rubber (EPDM) (typical unsaturated polyolefin rubber) as matrix because this material has been widely utilized in the fabrication of automotive tire sidewalls, cover strips, wires, cables, hoses, belting, footwear, roofing barriers, and sporting goods [11]. On the other hand, by utilizing the conventional rubber technology, we fabricated aligned carbon nanotube-based rubber sheet in a controllable direction. Up to my knowledge, this is the first report on the production of the aligned carbon nanotube-filled rubber even though there were a few reports on the alignment of carbon nanotubes in polymer using the magnetic fields [12-14].

\section{Experimental details}

High-purity and crystalline multi-walled carbon nanotubes (MWNTs) with diameter of ca. 100-200 nm were obtained by combining the synthesis of a catalytic CVD method $[8,9,15]$ and subsequent thermal heat treatment were synthesized in a large quantity. The formulation of matrix rubber (EP33, JSR Corp.) was summarized in Table 1.

Using a conventional laboratory Banbury mixer $\left(1800 \mathrm{~cm}^{2}\right)$, different amount of carbon nanotubes ( 5 and $30 \mathrm{wt} \%$ ) were mixed homogeneously in rubber matrix by controlling the mixing conditions (e.g., mixing time, mixing volume etc). Subsequently, by controlling the milling conditions (e.g., nip gap, mixing time, etc) of a conventional laboratory mill (200 $\mathrm{mm} \times$ $330 \mathrm{~mm}$ ), dispersion of carbon nanotubes in rubber matrix was accomplished by shear force. Then, we obtained CNTs aligned rubber sheet along the x-direction by applying calendaring and shaping process. In the case of CNTs-aligned rubber sheet along the z-direction, three step processes were carried out consecutively: extruding nanotube and rubber compounds into cylindrical shape, opening die of extruder for alignment of carbon nanotubes along the radial direction, and finally by opening it up. Finally, the experimental specimens were cured at $150{ }^{\circ} \mathrm{C}$ for 30 minutes.

The fractured surface was prepared by slicing the alignment direction of composite rubbers vertically with a microtome (Leica). Field-emission scanning electron microscope (FE-SEM) (5 $\mathrm{kV}$, a JEOL JSM-6335Fs) observations were carried out in order to confirm the dispersion and alignment of carbon nanotubes in rubber. The degree of orientation for CNTs was determined by wide angle X-ray diffraction (WAXD) method using $\mathrm{CuK}_{\alpha}$ radiation (Rigaku, RU-200B). Orientation coefficient $(f)$ of CNTs crystallites was calculated by using $(002)$ reflection at $2 \theta=$ ca. $27^{\circ}$ for CNTs by using equations described in ref. 16 . Tensile testing was performed with a 
universal testing machine (Instrong 5568) at an extension rate of $500 \mathrm{~mm} / \mathrm{min}$ at room temperature. Thermal conductivity (Thermolab-II, KES-F-M7, company), electrical conductivity (two-point method, company), and electromagnetic shielding characteristics (type, KEC method) of the samples were carried out.

\section{Results and discussion}

Carbon tubes used in this study exhibit relatively linear and long tubular morphology (high aspect ratio), as shown in Figure 1 (a) and (b). These tubes contain high crystallinity and relatively high real density which is paramount to conventional graphite. In addition, metallic impurity was detected in a level of below 100 part per million (measured by atomic absorption spectroscopy). Ideal status of nanotube alignment in the composites along the (a) X-and (b) Z-directions are illustrated in Figure 1 (c) and (d), respectively. In order to check dispersion of carbon nanotubes in rubber, fractured surfaces obtained by slicing the alignment direction of the rubber composite containing $30 \mathrm{wt} \%$ carbon nanotube vertically with a microtome were observed using the FE-SEM apparatus. As shown in Figure 1 (e) and (f), relatively long and straight nanotubes are protruded from the fractured surface. It is noteworthy that no aggregate of carbon nanotubes was observed. Therefore, it is possible to say that high-purity carbon nanotubes were homogeneously dispersed in rubber during conventional rubber processing (mixing and milling processes).

To evaluate the effect of nanotube on physical properties of rubber, mechanical property, thermal and electrical conductivities were measured as compared with neat rubber sheet and also those of boron nitride which is conventional filler used in rubber industry (see Table 2). The tensile at break of nanotube-added rubber is twofold higher than those of neat rubber sheet and boron-added rubber, accompanied without the deterioration of elongation break. The simple explanations for this mechanical property are (1) nano-sized effect of fibrous carbon (ca. $100 \mathrm{~nm}$ ), indicating highly increased interface area between filler and rubber when compared to boron nitride and (2) judicious alignment of fibrous carbons (high aspect ratio) (the degree of alignment of carbon nanotubes in rubber will be described using WAXD in detail). Furthermore, thermal conductivity of nanotube-added rubber was increased by at least a factor of 1.5 both directions, while volume resistivity of nanotube-added rubber was drastically lowered by six orders. It is assumed that the formation of conductive networks in rubber was formed, resulting in highly decreased resistivity of rubber sheet. In this sense, this rubber composite is highly applicable to antistatic, electromagnetic shielding, pressure sensor and actuators [17]. To study the mechanical properties of nanotube-added rubber composites in detail, stress-strain curves of 
$5 \mathrm{wt} . \%$ and $30 \mathrm{wt}$. \% nanotube-reinforced rubber sheet including neat rubber sheet were presented in Figure 2. For all samples, the initial modulus (below 10\% strain) is abruptly increased as the amount of nanotubes increases. In case of $5 \mathrm{wt}$ \% added rubber composites, where nanotubes are aligned along the $\mathrm{X}$-direction, elastic modulus for sample when force is applied to the alignment direction of carbon nanotubes is increased by ca. two times as compared with elastic modulus when vertically applied tensile force. But, with higher amount of nanotubes, the rubber composites show highly increased the initial modulus (stiff), but reduced tensile strength by ca. 0.5 and high elongation at break by ca. 0.4 , possibly due to increased cross-linking intensities.

It is well known that WAXD is one of powerful tools for characterizing the degree of crystallite of CNTs aligned in polymer matrix [12-14]. Figure 3 shows the X-ray diffraction photographs taken from different X-ray incident directions for the rubber composite filled with $30 \mathrm{wt} \% \mathrm{CNTs}$ aligned along the X-direction. This figure includes the azimuthal X-ray intensity profiles. The thinner white arrow indicates the Debye-Scherrer ring of the (002) plane of CNTs. Although the Debye-Scherrer ring is a circular form, the whiteness of the ring is more concentrated in the equatorial direction for Figure 3 (a) and (b), indicating the CNTs aligned in the X-direction as we expected. The degrees of orientation of CNTs crystallites in the composite is 0.60 , determined from azimuthal profiles of (a) and (b). In the case of the composite filled with $5 \mathrm{wt} \%$ CNTs, the selective orientation of CNTs also occurred in the directions we expected, but the degrees of orientation were considerably lower than that of $30 \mathrm{wt} \% \mathrm{CNTs}$ filled composite. The values are ca.0.54 for X-sheet, and 0.44 for Z-sheet, respectively, which means that the degrees of orientation for the composites containing $5 \mathrm{wt} \%$ CNTs are much lower that that containing $30 \mathrm{wt} \%$ CNTs. The difference between $5 \mathrm{wt} \%$ and $30 \mathrm{wt} \%$ CNTs filled composites may be attributed to the melt viscosity during the rubber milling processing. Since the melt viscosity with higher CNTs content is higher, the higher shear force may be generated during milling, which brings about higher orientation of CNTs. In addition, when filled $30 \mathrm{wt} \%$ CNTs, the higher viscosity may prevent the relaxation of aligned CNTs to random orientation, and retain the orientation of CNTs during solidification of rubber component.

Figure 4 shows the shielding characteristics of the nanotube-filled rubber sheet as compared with those of graphite particle- and ferrite-filled rubber sheets comparatively, as a function of frequency. The shield effect of the nanotube-filled rubber is prominent, especially in high-frequency bands of several hundred $\mathrm{MHz}$ or more. It is expected that the excellent shielding characteristics are mainly due to the formation of a conducting network in an insulating rubber matrix through the incorporation of the highly conductive carbon nanotubes. 
The demanded level of the shielding can be varied upon the environment. However, since the nanotube-filled rubber sheet exhibit excellent shielding effect above $60 \mathrm{~dB}$ or more in the frequency range of up to $1 \mathrm{GHz}$, we envisage that the nanotube-filled rubber sheet will be utilized in a wide range of electrical equipments.

\section{Conclusion}

We evaluated the mechanical, thermal, electrical and electromagnetic shielding properties of multi-walled carbon nanotubes incorporated EPDM rubber composite sheet. By varying the amount and alignment of nanotubes in a controllable direction, we can control the mechanical/electrical/thermal/shielding properties of nanotube/rubber sheet in detail, which is urgently required from the point of application view. Alignment of carbon nanotubes in rubber matrix resulted in significant improvements in elastic modulus (see Fig. 2), thermal and electrical conductivities, especially along the Z-direction. As the added amount of nanotubes is increased, rubber composites were transformed into a rigid material, which is reflected in a marked increase of the modulus. Based on wide angle diffraction study, it is sure that conventional rubber technology is quite useful for aligning carbon nanotubes in rubber in a controllable fashion. Furthermore, increased shear force (melt viscosity) caused by the higher amount addition of nanotube (30 wt. \%) during the milling process give rise to improved alignment of carbon nanotubes in rubber matrix. Therefore, it is envisaged that judicious selection of amount, alignment of carbon nanotubes in rubber make us applicable to various fields.

\section{Acknowledgements}

This work was supported by the CLUSTER of Ministry of Education, Culture, Sports, Science and Technology.

\section{References}

[1] Dresselhaus MS, Dresselhaus G, Eklund PC. Science of Fullerenes and Carbon Nanotubes, Academic Press; San Diego, 1996:756-864

[2] Iijima S. Nature 1991;354:56.

[3] Oberlin A, Endo M, Koyama T J Crys Grow 1976;32:335-349.

[4] Baughman RH, Zakhidov AA, de Heer WA. Science 2002;297:787-792. 
[5] Treacy M, Ebbesen TW, Gibson JM. Nature 1996;381:678-89.

[6] Dai H, Wong EW, Lieber CM Science 1996;272:523-56

[7] Ebbesen TW, Lezec H J, Hiura H, Bennett JW, Ghaemi HF, Thio T Nature 1996;382:54-56.

[8] Endo M. Chem. Tech. 1998;18:568-576

[9] Endo M, Kim YA, Fukai Y, Hayashi T, Terrones M, Terrones H, et al. Appl Phys Lett 2001; 79:1531-1534.

[10] Thostenson ET, Ren Z, Chou TW. Composites Science and Technology 2001;61:1899-1912, and references therein.

[11] Ciesielski A. An Introduction To Rubber Technology, Rapra Technology Ltd., 1999.

[12] Kimura T, Ago H, Tobita M, Ohsima S, Kyotani M, Yumura M. Advanced Materials 2002;14:1380-1383.

[13] Garmestani H, Al-Haik MS, Dahmen K, Tannenbaum R, Li D, Sablin SS, et al. Advanced Materials, 2003, 15, 1918-1921

[14] Takahashi T, Yonetake K, Koyama K, Kikuchi T, Macromol Rapid Commun 2003;24: 763-767.

[15] Endo M, Kim YA, Hayashi T, Nishimura K, Matushita T, Miyashita K, et al. Carbon 2001; 39:1287-1297.

[16] Heuvel HM, Huisman R, Lind KCJB. Journal of Polymer Science: Polymer Physics 1976:921-940.

[17] Donnet JB, Bansal RC, Wang MJ. Carbon Black, second ed., Marcel Dekker Inc., New York, 1993

\section{Figure Captions}

Figure 1 (a), (b) FE-SEM images of highly crystalline carbon nanotubes at different resolutions. Scale bars: $1 \mu \mathrm{m}$; (c), (d) Schematic images of carbon nanotube incorporated rubber sheets along the X- and Z-directions, respectively; (e) and (f) FE-SEM images of CNTs30/rubber composites showing high dispersion of carbon nanotubes along the aligned directions. It is noteworthy that there was no aggregated in rubber matrix, indicating good dispersion of carbon nanotubes.

Figure 2 Stress-strain curves of aligned carbon nanotube-filled rubber composites including neat rubber sheet. 
Figure $3 \mathrm{X}$-ray photographs and azimuthal X-ray intensity profiles for $30 \mathrm{wt} \%$-CNTs filled rubber composites; X-ray incident directions are (a) Z, (b) Y, and (c) X.

Figure 4 Frequency dependence of EMI shielding for carbon nanotube-, graphite particle- and ferrite-filled rubber sheets at the same 30 weight percents. 